\title{
cGMP-Dependent Protein Kinase Encoded by foraging Regulates Motor Axon Guidance in Drosophila by Suppressing Lola Function
}

\author{
Qionglin Peng, ${ }^{1,2 *}$ Yijin Wang, ${ }^{1,2 *}$ Meixia Li, ${ }^{1 *}$ Deliang Yuan, ${ }^{1,2}{ }^{\circledR}$ Mengbo Xu, ${ }^{1}$ Changqing Li, ${ }^{1}$ Zhefeng Gong, ${ }^{1}$ \\ Renjie Jiao, ${ }^{1}$ and $\mathrm{Li} \mathrm{Liu}^{1,2,3}$ \\ ${ }^{1}$ State Key Laboratory of Brain and Cognitive Science, Institute of Biophysics, Chinese Academy of Sciences, Beijing 100101, China, ${ }^{2}$ University of the \\ Chinese Academy of Sciences, Beijing 100039, China, and ${ }^{3}$ Key Laboratory of Mental Health, Chinese Academy of Sciences, Beijing 100101, China
}

Correct pathfinding and target recognition of a developing axon are exquisitely regulated processes that require multiple guidance factors. Among these factors, the second messengers, cAMP and cGMP, are known to be involved in establishing the guidance cues for axon growth through different intracellular signaling pathways. However, whether and how cGMP-dependent protein kinase (PKG) regulates axon guidance remains poorly understood. Here, we show that the motor axons of intersegmental nerve $b$ (ISNb) in the Drosophila embryo display targeting defects during axon development in the absence of foraging ( for), a gene encoding PKG. In vivo tag expression revealed PKG to be present in the ventral nerve code at late embryonic stages, supporting its function in embryonic axon guidance. Mechanistic studies showed that the transcription factor longitudinal lacking (lola) genetically interacts with for. PKG physically associates with the LolaT isoform via the C-terminal zinc-finger-containing domain. Overexpression of PKG leads to the cytoplasmic retention of LolaT in S2 cells, suggesting a role for PKG in mediating the nucleocytoplasmic trafficking of Lola. Together, these findings reveal a novel function of PKG in regulating the establishment of neuronal connectivity by sequestering Lola in the cytoplasm.

Key words: Drosophila; Lola; motor axon guidance; PKG

Significance Statement

Axon pathfinding and target recognition are important processes in the formation of specific neuronal connectivity, which rely upon precise coordinated deployment of multiple guidance factors. This paper reveals the role of cGMP-dependent protein kinase (PKG) in regulating the pathfinding and targeting of the developing axons in Drosophila. Moreover, our study indicates that PKG regulates the cytoplasmic-nuclear trafficking of the transcription factor LolaT, suggesting a mechanism of PKG in directing motor axon guidance. These findings highlight a new function of PKG in axon guidance by suppressing a transcription factor.

\section{Introduction}

The formation of specific connections between neurons and their targets is essential for establishing a functional nervous system.

Received Oct. 10, 2015; revised March 15, 2016; accepted March 21, 2016.

Author contributions: Q.P., C.L., Z.G., R.J., and L.L. designed research; Q.P., Y.W., M.L., D.Y., and M.X. performed research; Q.P., R.J., and L.L. analyzed data; Q.P., R.J., and L.L. wrote the paper.

This work was supported by the Strategic Priority Research Program B of the Chinese Academy of Sciences (XDB02040002), the Ministry of Science and Technology of China (2012CB825504), and the National Natural Sciences Foundation of China (31030037, 31571033, 81470846, 31271573, 31529004). We thank Prof. Yongqing Zhang for providing the flies; Prof. Edward Giniger for the gift of Lola antibody; Haiyun Gong, Junying Jia, and Xudong Zhao of the IBP core facility center for technical assistance; Bloomington Drosophila Stock Centers; Vienna Drosophila RNAi Center; and Drosophila Genetic Resource Center at Kyoto Institute of Technology.

The authors declare no competing financial interests.

${ }^{*}$ Q.P., Y.W., and M.L. contributed equally to this work.

Correspondence should be addressed to either Dr. Renjie Jiao or Dr. Li Liu, State Key Laboratory of Brain and Cognitive Science, Institute of Biophysics, Chinese Academy of Sciences, Beijing 100101, China. E-mail: rjia0@sun5.ibp.ac.cn or liuli@sun5.ibp.ac.cn.
The development of these connections is determined, at least in part, by selective choices made by growing axons, which are directed by different guidance factors, including transmembrane receptors, intracellular signaling molecules, and transcription factors (Dickson, 2002; Kolodkin and Tessier-Lavigne, 2011). The guanylyl cyclase (GC) Gyc76C is required in motor neurons for Sema-1a-PlexA-mediated repulsive axon guidance in Drosophila (Ayoob et al., 2004; Chak and Kolodkin, 2014). Intracellular second messengers, cAMP and cGMP, determine the direction of growth cone steering by modulating calcium channels (Song et al., 1998; Nishiyama et al., 2003). These two mes-

Y. Wang's present address: Neuroscience Research Institute and Department of Molecular, Cellular, and Developmental Biology, University of California, Santa Barbara, CA 93106.

Z. Gong's present address: Department of Neurobiology, Zhejiang University School of Medicine, Hangzhou, Zhejiang 310058, China.

DOI:10.1523/JNEUROSCI.3726-15.2016

Copyright $\odot 2016$ the authors $\quad 0270-6474 / 16 / 364635-12 \$ 15.00 / 0$ 
sengers also direct the formation of axons and dendrites in cultured hippocampal neurons through the regulation of protein kinases (Shelly et al., 2010). These findings suggest that cAMP and cGMP signaling pathways are crucial during the development of the nervous system.

As a key component of the cGMP signaling pathway, PKG is well known for its functions in cardiac protection, smooth muscle relaxation, neuronal plasticity, and learning and memory (Kaun et al., 2007; Wang et al., 2008; Francis et al., 2010; Kohn et al., 2013). However, little is known about the role of PKG in axon guidance. A previous study showed that, in the absence of cGMPdependent protein kinase I (PKG I), the trajectories of sensory axons in the spinal cord of mouse embryos extend predominantly in a rostral direction, whereas in wild-type mice they point equally in both rostral and caudal directions (Schmidt et al., 2002). In Drosophila, PKG is encoded by the foraging ( for) gene, two variations of which, for ${ }^{R}$ (Rovers) and for ${ }^{S}$ (Sitters), have been identified according to dimorphic larval foraging strategies (Osborne et al., 1997). Interestingly, the Sitter larvae, which have lower PKG activity, show increased ectopic nerve endings in neuromuscular junctions and increased transmitter release after nerve stimulation in focal recordings compared with the Rover larvae (Renger et al., 1999). However, the exact role of PKG in axon guidance and in modulating growth cone responses remains to be elucidated.

Transcription factors are required to regulate neural diversity and wiring specificity (Zarin et al., 2014). The longitudinal lacking (lola) gene encodes a number of transcription factors with conserved N-terminal BTB domains and variable C-terminal zincfinger domains. Splicing variants of lola show distinct expression patterns (Goeke et al., 2003; Horiuchi et al., 2003), which function in a wide range of developmental processes, including axon guidance, neural specification, and tumorigenesis (Giniger et al., 1994; Madden et al., 1999; Goeke et al., 2003; Ferres-Marco et al., 2006; Spletter et al., 2007). lola mutants exhibit defects of axon growth and guidance in both the CNS and PNS of Drosophila embryos (Giniger et al., 1994). In the CNS, Lola is required for repelling longitudinal axons away from the midline through augmenting the expression of the midline repellant Slit and axonal receptor Robo (Crowner et al., 2002). In the PNS, Lola promotes ISNb axon growth, in part by suppressing the expression of an actin nucleation factor, spire, and controls synapse formation through regulating the expression of glutamate receptors (Gates et al., 2011; Fukui et al., 2012).

In this study, by observing the axon development of ISNb, we show that neuronal PKG is required for axon pathfinding and target recognition in the embryonic PNS of Drosophila. In addition, $\mathrm{PKG}$ is also involved in axon guidance in the CNS, suggesting a general role of PKG during the development of the entire nervous system. Further, we show that for and lola genetically interact with each other during motor axon guidance; and at the molecular level, PKG antagonizes the function of Lola by sequestering Lola in the cytoplasm, preventing Lola from entering the nucleus to regulate transcription.

\section{Materials and Methods}

Fly strains and genetics. All flies of either sex used in this study were raised on standard corn food at $25^{\circ} \mathrm{C}$ and in $60 \%$ humidity with a $12 \mathrm{~h}$ light $/ 12 \mathrm{~h}$ dark cycle (Guo et al., 1996). $w^{1118}$ was used as a control strain. Lethal mutants were maintained over a $C y O$, armGFP balancer. elav-Gal4, 24B-Gal4, $D f(2 L) E D 243$, and UAS-lola RNAi lines were obtained from the Bloomington Stock Center (Indiana University, Bloomington, Indiana). NP285-Gal4 was from the Drosophila Genetic Resource Center (Kyoto Institute of Tech- nology, Kyoto, Japan). UAS-for RNAi [ 138320 ] was from the Vienna Drosophila RNAi Center (Vienna). EP-lola (EP2537) and other EP lines for the genetic screen were from Y. Zhang (Institute of Genetics and Developmental Biology, Beijing, Chinese Academy of Sciences). for null mutant ( for ${ }^{20-29}$ ), lolaT mutant (lola $T^{38-1}$ ), and for- $v 5$ in vivo tagged lines were generated by CRISPR/Cas9-mediated gene targeting. gRNA design conformed with the target sequence principle: $5^{\prime}$-GG-N $\mathrm{N}_{17-19}-\mathrm{NGG}-3^{\prime}$, in which NGG stands for the small protospacer-adjacent motif (PAM) (Yu et al., 2014). The binding sequences of gRNAs used in this study were as follows: gRNA-for ${ }^{20-29}: 5^{\prime}$ GGACGATTCTACGGGACAAGGG-3', gRNA-lolaT ${ }^{38-1}: 5^{\prime}$-GGCATCT TCGATACGAGTCTGG-3', gRNA-for-v5: 5'-GGCGGACCCTCAGG ATCGGGAGG-3'. Cas9 mRNAs and gRNAs were transcribed in vitro. The prepared injection mixtures were injected into $w^{1118}$ embryos according to a previously described protocol (Yu et al., 2014). pBluescript KS was used to generate the donor plasmid for the in vivo tagged for-v5 line. The left homologous arm (HA-L, $1.8 \mathrm{~kb}$ ) with a $v 5$-loxP sequence and the right homologous arm (HA-R, $1.4 \mathrm{~kb}$ ) were PCR-amplified using Lig $4^{169}$ genomic DNA as template before cloning into pBluescript KS. The $v 5$-loxP sequence was directly added to the end of the primer and was amplified together with the left homologous arm. The mixture containing Cas9 mRNAs, gRNAs, and donor plasmid was microinjected into the lig $4^{169}$ embryos. The lig $4^{169}$ genetic background was later changed to $w^{1118}$ by crossing. The primers used for amplifying HAs were as follows: for-HA-L-forward: 5'-ATAGCGGCCGCGTG GCGACTACATAGTGCGC-3', for-HA-L-reverse: 5'-ACCAAGCT TTCACGTAGAATCGAGACCGAGGAGAGGGTTAGGGATAGGCTT ACCGGATAACTTCGTATAATGTATGCTATACGAAGTTATGAAGTC CTTGTCCCATCCAG-3', for-HA-R-forward: 5'-ACCAAGCTTGGAGAA TCAGAACCCGTT-3', for-HA-R-reverse: 5'-CCACTCGAGCCA CGACTCACCTATTATC- $3^{\prime}$. The following PCR primers were used for the identification of mutant lines and for the in vivo tagged for- $v 5$ line: for ${ }^{20-29}$ forward: 5' -CTCGAACATGAGCACTCC-3' for $^{20-29}$-reverse: $5^{\prime}$-AACCTCACAGATCTGCGC-3', lola $^{38-1}$-forward: 5' -CTCCTGGACGATATC CGTG-3', lola $T^{38-1}$-reverse: $5^{\prime}$-GTGTTTGCTGTACAGCAC-3', for-v5forward: 5'-GGAGGAGACGCACTACCAGC-3', for- 25 -reverse: 5' CTTTGGCTGTCTGCAGGCTG-3'.

The UAS-forP2 transgenic line was constructed with a full-length cDNA of the forP2 coding sequence and used in the previous study (Wang et al., 2008). UAS-for RNAi [2dsf07] and UAS-myc-lolaT transgenic lines were generated using previously described protocols (Wang et al., 2008). The $6 \times$ Myc tag (EQKLISEEDL) was added to the $\mathrm{N}$ terminus of the LolaT protein. The RNAi fragments of for and the full-length sequence of lolaT were amplified from wild-type cDNAs using the following primers: for RNAi [2dsfo7]forward: 5'-CGGGGATCCGGAGTTGGAGGCTTCGGTC-3', for RNAi [2dsf07]-reverse: 5'-CGGGGTACCTAATCCGCACTGATGTCGTG-3', lolaT-forward: 5'-ATAGCGGCCGCATGGATGACGATCAGC-3', lola T-reverse: 5'-CCGTCTAGACTAGTTATTAAGGTAATC-3'. All constructs were verified by sequencing.

Immunostaining and imaging. Embryos at late Stage 16 were collected, dissected, fixed, and stained for ISNb according to previously described methods (Lee et al., 2009). Staining of other embryos was performed according to a common method (Patel, 1994). Drosophila S2 cells were cultured at $25^{\circ} \mathrm{C}$ in serum-free insect cell culture medium (HyClone \#SH30278.02). For S2 cell staining, $2 \mathrm{ml}$ cell cultures in 6-well culture dishes were transfected with appropriate plasmids using Lipofectamine 3000 Reagent (Invitrogen \#L3000-008). Forty-eight hours after transfection, S2 cells were moved onto poly-lysine-coated coverslips and incubated for another $1 \mathrm{~h}$. Subsequently, cells were fixed with $4 \%$ formaldehyde for $20 \mathrm{~min}$, permeabilized, and blocked with $0.1 \%$ Triton X-100 in PBS containing 10\% normal goat serum for $30 \mathrm{~min}$ at room temperature. Cells were then incubated with the primary antibody followed by the secondary antibody. The following antibodies were used for immunostaining: mouse anti-FasciclinII/mAb 1D4 (1:100; Developmental Studies Hybridoma Bank), mouse mAb BP102 (1:100; Developmental Studies Hybridoma Bank), 488 phalloidin (1:400; Invitrogen \#A12379), mouse anti-V5 (1:100; Invitrogen \#R960-25), FITCconjugated goat anti-HRP (1:400, Jackson ImmunoResearch Laboratories \#123-095-021), rabbit anti-Lola (1:50, a gift from Prof. Edward Giniger), rabbit anti-Flag (1:500; Sigma \#F7425), and mouse anti-Myc (1:500; B\&M Biotech \#M047-3). Fluorescent secondary antibodies were 
used for signal detection. DNA was visualized with $0.5 \mathrm{mg} / \mathrm{ml}$ DAPI. Embryos or S2 cells were mounted in Vectashield (Vector Labs H-1000). Stacks of images were obtained using a Leica SP8 or SP5 confocal microscope. Images were processed using Image (National Institutes of Health, version 1.42).

In the genetic screen, 3-d-old flies of either sex in correct genotype were examined and counted. Images of wings from male flies were captured by a Leica MZ16F stereo fluorescence microscope. Images of eyes from female flies were obtained using Hitachi TM3000 tabletop microscope.

Cell synchronization by exposure to hydroxyurea. Transfected S2 cells, cultured in 6-well plates for $30 \mathrm{~h}$, were treated with $1 \mathrm{~mm}$ hydroxyurea for another $18 \mathrm{~h}$ (Heinemann et al., 2010). The cells were harvested, fixed, and stained with propidium iodide. Normal cycling control samples without treatment with $1 \mathrm{~mm}$ hydroxyurea were collected at the same time and processed in the same manner. Cells were assessed using flow cytometry (BD Biosciences FACS Calibur) to determine DNA content and cell cycle phase.

Generation of expression constructs. Full-length sequences of for and lola, amplified from wild-type cDNAs, were cloned into pAC5.1-A. The $3 \times$ Flag (DYKDDDDK) and $6 \times$ Myc tags were added to the $\mathrm{N}$ termini of PKG and Lola proteins, respectively. Truncated lolaT fragments, encoding the $\mathrm{N}$ terminus containing the conserved BTB domain (amino acids 1-454) and the variable $\mathrm{C}$ terminus containing the zinc fingers (amino acids 455-576) were also separately cloned into pAC5.1-A. Later, a strong, constitutive NLS (PKKKRKV) was added to the $\mathrm{C}$ termini of Myc-LolaT proteins. The primers used for the construction of pAC5.1-myc-lolaT were the same as those used in pUAST-myc-lolaT. Three different isoforms of Lola (A, L, T) share the same forward primer. Other primers used for the constructions were as follows: forP2-forward: 5'-ATAGCGGCCGCATGCAGAGTCTGCG GATCTCG-3' , forP2-reverse: 5'-AGTACCGGTCCGCGGTCAGAAGTC CTTGTCCCATCC-3, lolaA-reverse: 5' -CCGTCTAGACTACAATCCC TCCCCG-3', lolaL-reverse: 5' -CCGTCTAGACTAAAACAAATATTT GG-3', lola-N-forward: 5'-ATAGCGGCCGCATGGATGACGATCA GC-3', lola-N-reverse: 5'-CCGTCTAGACTATTGGGGATCCCGTTGC3', lolaT-C-forward: 5'-ATAGCGGCCGCATGGAAAACTCCTGGACG3', lolaT-C-reverse: 5'-CCGTCTAGACTAGTTATTAAGGTAATC-3', lolaT-NLS-forward: 5' -TCTGCGGCCGCATGGATGACGATC-3', lola T-NLS-reverse: 5' -ATATCTAGACTACACCTTCCTCTTCTTCTTGGGG TTATTAAGGTAATCAAG-3' . All constructs were verified by sequencing.

Coimmunoprecipitation. Total protein extracts from Drosophila S2 cells or Stage 16 embryos were prepared in lysis buffer $(20 \mathrm{~mm}$ Tris- $\mathrm{HCl}$, pH 7.5, 150 mм NaCl, 1 mм EDTA, pH 7.4, 0.5\% Triton X-100, 10\% glycerol) in the presence of protease inhibitors [cOmplete, Mini (Roche \#4693124001), PhosStop (Roche \#4906845001)]. For coimmunoprecipitation, lysates were incubated with tag-conjugated agarose beads [mouse anti-Flag-beads (Sigma \#A2220) or mouse anti-Myc-beads (Abmart \#20012)] overnight at $4^{\circ} \mathrm{C}$. After washes in lysis buffer, immunoprecipitates were boiled in $6 \times$ SDS loading buffer for dissociation from beads. Rabbit anti-Flag (1:1000, Sigma \#F7425), rabbit anti-Myc (1:5000, B\&M Biotech \#562), rabbit anti-PKG (1:1000), and mouse anti-tubulin (1:2000) were used for Western blots to detect coimmunoprecipitated proteins.

Generation of anti-PKG antibody. Polyclonal anti-PKG antibody was generated using the synthetic peptide, CKPAVKSVVDTTNFDDYP, as antigen. The peptide was used to immunize a rabbit for anti-PKG serum production. The anti-PKG antibody was affinity purified (B\&M Biotech).

Statistical analyses. For the quantification of ISNb motor axon defects, A2-A6 segments of each embryo at late Stage 16 were chosen for analysis. ISNb axon defects were defined as mistargeting with improper projections to ventral muscles or abnormal associations with adjacent motor axons, which were only very rarely observed in control $\left(w^{1118}\right)$. Other defects, such as stalled, bypass, and premature defasciculation, were rarely detected in either mutants or control $w^{1118}$ embryos. For the quantification of the midline crossing defects in CNS, six abdominal segments were analyzed per embryo. In the genetic screen, the blistered or normal wings of the flies with correct genotypes were counted and calculated for the rescue percentages of balloon wings. For the quantification of the percentage of the cells with cytoplasmic retention of Lola proteins, trans- fected S2 cells in normal size, excluding cells in M phase, which are easily recognized by chromosome feature, were counted. Fisher's exact test was performed to analyze the percentages in different circumstances.

\section{Results}

\section{Loss of for function leads to developmental defects in ISNb motor axon pathfinding and target recognition}

We have previously shown that the for gene is involved in visual pattern memory in the central complex of Drosophila (Wang et al., 2008). To investigate further whether for is also involved in neural development, we first examined ISNb motor axons at late embryonic Stage 16 in a for deficiency line, $D f(2 L) E D 243$ (Ryder et al., 2004), referred to here as for ${ }^{D f(2 L) E D 243}$, which carries a 24.7 $\mathrm{kb}$ deletion at the for gene genomic locus (Belay et al., 2007). In wild-type embryos, ISNb axons first defasciculate from the ISN bundle and extend to the ventral longitudinal muscles, then defasciculate at distinct points to establish three initial presynaptic contacts with the target muscles 6, 7, 12, and 13 (Landgraf et al., 1997). In homozygous for ${ }^{D f(2 L) E D 243}$ mutant embryos, ISNb axons often display targeting defects, such as muscle target recognition errors or abnormal associations with other motor axons (data not shown).

To confirm the novel function of for in ISNb motor axon guidance described above, we generated a null allele, for ${ }^{20-29}$, using the CRISPR/Cas9 system. The gRNA-binding site was chosen within the kinase domain that is conserved in all PKG isoforms (Fig. 1A). The for ${ }^{20-29}$ null mutant contains a 1 bp deletion that results in a frame shift in all PKG isoforms (Fig. 1A). Compared with the control (Fig. $1 B$ ), homozygous for ${ }^{20-29}$ mutant embryos display overgrowth or turning to target incorrect muscles, resulting in ectopic nerve endings (Fig. $1 C, D$, arrows) and occasionally abnormal associations with adjacent axons (Fig. 1E, arrows). The schematic diagrams showing the wild-type ISNb axons and the mistargeting defects are presented in Figure $1 F$. The percentages of total ISNb defects or mistargeting defects in both for ${ }^{D f(2 L) E D 243}$ and for ${ }^{20-29}$ null mutants are significantly different from that of the $w^{1118}$ control (Fig. $1 G$ ), whereas the incidence of ISNb defects between the two kinds of for mutants is not significantly different. These results indicate that for is involved in regulating axon pathfinding and target recognition during embryonic development in Drosophila.

\section{Neuronal for is required for ISNb motor axon pathfinding}

Next, we wondered whether the defects we observed in motor axon pathfinding and target recognition result from the loss of for function in the muscles or the neurons, or both. As shown in Figure $1 B-E$, the pattern and morphology of the embryonic ventrolateral muscles are normal in for mutant line compared with $w^{1118}$. Importantly, the total defects and mistargeting defects of ISNb axons in for ${ }^{D f(2 L) E D 243}$ homozygous embryos were not rescued by overexpression of forP2, an untagged full-length transcript of for gene that we have previously described (Wang et al., 2008), under the control of a pan-muscle Gal4 driver, 24B-Gal4 (Fig. 1G), whereas the total defects and mistargeting defects of ISNb axons in for ${ }^{D f(2 L) E D 243}$ or for ${ }^{20-29}$ were both partially rescued by pan-neuronal expression of PKG-P2 driven by elav-Gal4 (Fig. $1 G$ ).

To substantiate that neuronal PKG contributes to ISNb motor axon pathfinding and target recognition, we used RNAi transgenes to knock down the expression of for. When neuronal for was knocked down by the expression of for RNAi [2dsf07] or for $R N A i$ [v38320] driven by elav-Gal4, the ISNb axons showed significantly increased total defects and similar mistargeting defects to those observed in the for mutants compared with the control 
A

\begin{tabular}{rrrr}
$3622 \mathrm{~K}$ & $3623 \mathrm{~K}$ & $3624 \mathrm{~K}$ & $3625 \mathrm{~K}$ \\
\hline
\end{tabular}

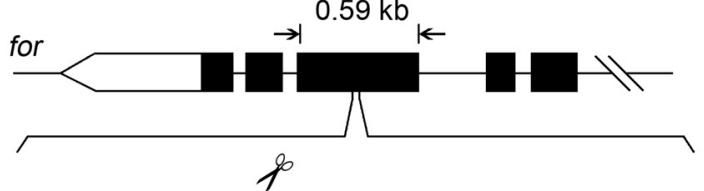

WT: $\quad 3{ }^{\prime}$-TTCAACGGGAACAGGGCATCTTAGCAGGTCTCGA-5'

for ${ }^{20-29}: 3^{\prime}$-TTCAACGGGAAC • GGGCATCTTAGCAGGTCTCGA-5'

$$
\text { go Cas9 }
$$

NGG PAM sequence

- Deleted sequence gRNA binding site
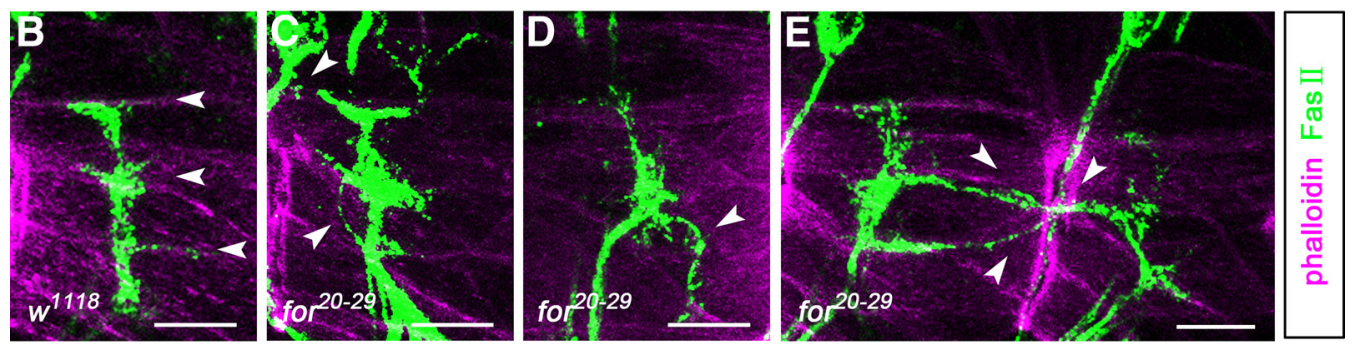

$\mathbf{F}$
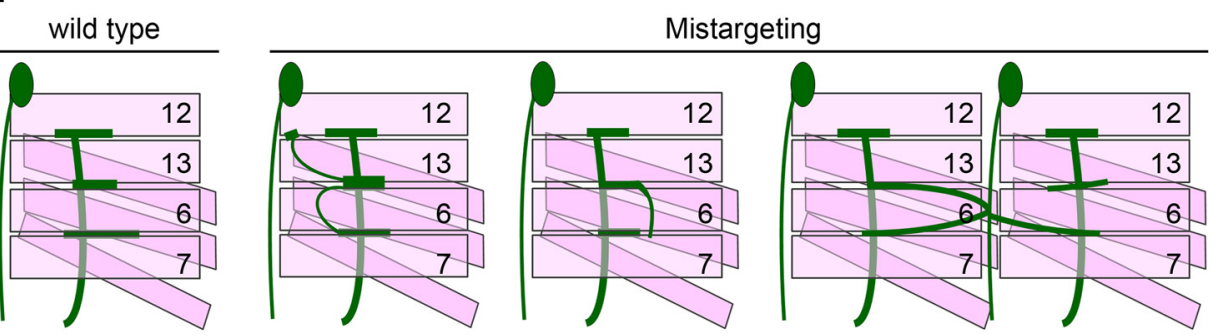

G

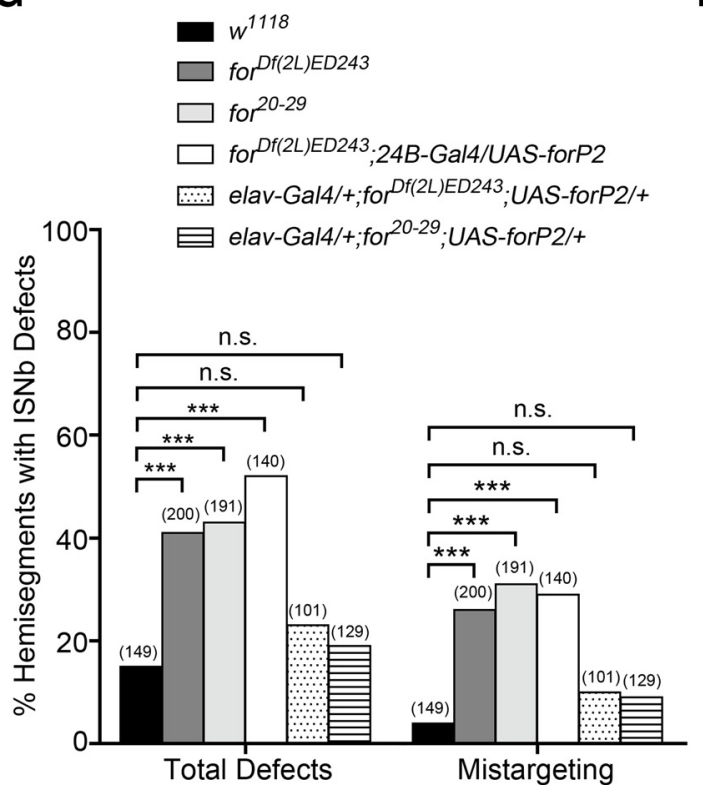

H
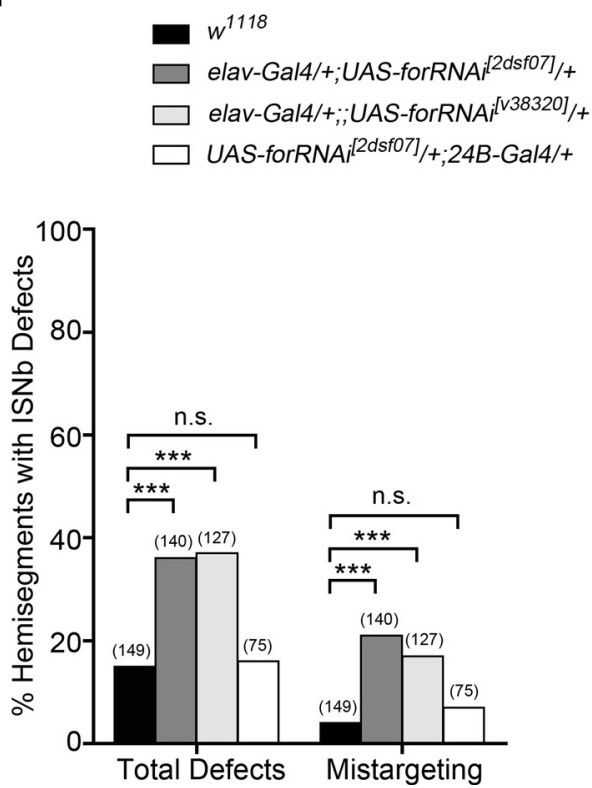

Figure 1. for is required for proper ISNb motor axon pathfinding and target recognition. A, Schematic diagram showing the strategy of generation and characterization of a for ${ }^{20-29}$ null mutant using the CRISPR/Cas9 system. Scissors indicate where the Cas9 cleaves at the for locus. The PAM sequence is marked in red. The gRNA binding sequence is underlined. Red dot represents the $1 \mathrm{bp}$ deletion in the for genomic sequence. $\boldsymbol{B}-\boldsymbol{E}$, ISNb motor axon projections of late Stage 16 embryos were visualized with anti-Fasll (green). Muscle was stained with phalloidin (magenta). All panels, Anterior is to the left and dorsal is top. Scale bar, $10 \mu \mathrm{m} . B, \ln w^{1118}$ embryos, ISNb axons navigate dorsally and defasciculate when they arrive between muscles 6 and 7 , at the proximal edge of muscle 13 , and innervate appropriate muscles at muscle 12 (arrows). $\boldsymbol{C}, \boldsymbol{D}$, In for ${ }^{20-29}$ null mutant embryos, ISNb axons follow aberrant pathways and target improper muscles (arrows). $\boldsymbol{E}$, In for ${ }^{20-29}$ null mutants, ISNb axons in two neighboring segments abnormally associated (arrows). $\boldsymbol{F}$, Schematic diagrams showing normal and defective ISNb pathways. $\boldsymbol{G}$, Quantification of total defects and mistargeting ISNb phenotypes in $w^{1718}$, fo ${ }^{\text {Df(2L)ED243 }}, \mathrm{for}^{2^{2-29}}$ and rescued embryos. H, Quantification of total defects and mistargeting ISNb phenotypes of embryos in which endogenous for was knocked down in either all neurons or all muscle cells driven by elav-Gal4, or 24B-Gal4, respectively. Knockdown of for in the nervous system leads to a significant increase of ISNb mistargting defects compared with the $w^{1118}$ control. The number of abdominal hemisegments scored for each genotype is shown in parentheses. ${ }^{* * *} p<0.001$ (Fisher's exact test). n.s., Not significant. 
embryos (Fig. 1H). The two RNAi lines used in this study are both for all transcripts of the for gene, which were validated by real-time RT-PCR to ensure the knockdown efficiency (data not shown). Knockdown of for in all muscles by $24 B$-Gal4-driven RNAi resulted in no significant ISNb total defects and mistargeting defects (Fig. 1H). These results indicate that neuronal PKG, rather than muscular PKG, is responsible for normal ISNb motor axon pathfinding and target recognition during development.

\section{for is expressed in the embryonic ventral nerve cord (VNC)}

PKG is widely localized in the adult brain, but mostly in neurons (Belay et al., 2007). To support the observation that for is involved in ISNb targeting during embryonic development, we expected PKG to be present in the embryonic nervous system. To investigate this, a molecular tag was fused to the for coding region in vivo, designated for-v5, using the CRISPR/Cas9 system. This genetically modified fly line was used to examine the expression pattern of PKG at embryonic stages. For the in vivo tagging of for with V5, a gRNA-binding sequence was chosen before the stop codon of the for gene (Fig. 2A). The donor plasmid for V5 insertion containing the HA-L, the fused $v 5$-loxP fragment, and the HA-R sequences is schematically shown in Figure 2A. After homologous recombination (HR), successful insertion of $\mathrm{V} 5$ was detected by PCR using primers corresponding to the inserted V5 sequence and the for genomic sequence (Fig. 2B). Genomic DNA of for- $v 5$ flies was sequenced to ensure a clean "endsout" recombination. PKG-V5 fusion proteins were extracted from for- -15 embryos and detected by Western blotting using an anti-V5 antibody (Fig. 2C).

Next, we examined the localization pattern of PKG in for- $v 5$ embryos using an anti-V5 antibody. We observed that PKG is ubiquitously expressed throughout the embryonic stages; however, a specific PKG pattern was detected in late embryonic stages. To make a better presentation of the cells with high expression levels of PKG, limited $Z$-stacks of the staining results were chosen and processed. At the late embryonic Stage 16, PKG displays a segmental distribution and is enriched in small sets of cells in the VNC (Fig. 2D,E). However, almost all other cells have low expression levels of PKG. To explore whether those cells with high expression levels of PKG are neurons, we used elav-Gal4 to express a nuclear GFP (GFP.nls) in neurons in the for- $v 5$ genetic background. PKG-V5 was detected with a high level in the cytoplasm of the neurons (Fig. $2 \mathrm{~F}-\mathrm{H}$ ) located in the midline of the VNC.

To determine whether PKG is also involved in axon guidance in the CNS, we first used a BP102 antibody to detect CNS axons in for ${ }^{D f(2 L) E D 243}$ mutants at embryonic late Stage 16 . We found that the areas between anterior and posterior commissures in each segment of for ${ }^{D f(2 L) E D 243}$ mutants were smaller than those of $w^{1118}$ embryos (data not shown). To further confirm the CNS axonal defects in for mutants, we examined CNS axons in for ${ }^{D f(2 L) E D 243}$ mutants using HRP and anti-FasI antibody at the same time. In $w^{1118}$ embryos, CNS axons exhibit a regular axonal scaffold organization detected by HRP, or three parallel longitudinal axon tracts along each side of the midline detected by anti-FasII antibody (Fig. 2I-K). As we expected, the CNS axonal defects in for ${ }^{D f(2 L) E D 243}$ mutants are associated with the midline crossing (Fig. $2 L-N$; Fig. $2 N$, arrows), which were also partially rescued by pan-neuronal expression of for $\mathrm{P} 2$ driven by elavGal4 (Fig. 2O). These results suggest that PKG is required for axon guidance in both the PNS and CNS of Drosophila embryos.

\section{Genetic interaction between for and lola}

To investigate the mechanism by which PKG regulates ISNb axon pathfinding and target recognition, we looked for genes that genetically interact with for. A yeast two-hybrid study, in which 10,623 predicted full-length transcripts in Drosophila were isolated and screened, has reported that PKG physically interacts with several proteins, including Lola and several other proteins (Giot et al., 2003). However, it remains unknown which protein isoforms or domains are responsible for the interaction. To confirm that whether these genes have genetic interaction with for gene, and to find out other candidate genes, we performed a genetic screen using a different system from the embryonic PNS based on our previous finding that PKG regulates the development of the wing and the eye. When PKG is overexpressed under the control of the for regulatory elements (NP285-Gal4), flies display a balloon wing phenotype compared with the normal wings (Fig. $3 A, B$ ). Using this balloon wing phenotype as a read out, we screened $349 \mathrm{EP}$ (enhancer and promoter) lines for genes, in which gain-of-function mutations suppressed the phenotype. Eight candidate genes were identified, also including the transcription factor lola. We found that overexpression of lola partially rescued the balloon wing defects induced by the overexpression of forP2 (Fig. 3C-E). In another test system, knockdown of for in the eye of flies, driven by GMR-Gal4, causes a rough eye phenotype, which can be completely suppressed by a simultaneous knockdown of all transcripts of lola (Fig. 3F-M). These genetic results suggest that for antagonizes the function of lola in different tissues; we thus hypothesized that for and lola may also genetically interact in the process of ISNb axon guidance. As described in the above sections, for ${ }^{D f(2 L) E D 243}$ mutants show ISNb targeting defects during axon guidance (Fig. $3 N$ ). As expected, knockdown of lola transcription in the nervous system rescued the ISNb targeting defects of for ${ }^{D f(2 L) E D 243}$ homozygous embryos, although this rescue was only partial (Fig. 3O,P). The expressivity of the for ${ }^{D f(2 L) E D 243}$ phenotype was reduced from $26 \%$ to $10.42 \%$ for ISNb mistargeting defects, and from $40.5 \%$ to $23.96 \%$ for ISNb total defects (Fig. 3Q). Together, these results indicate a genetic interaction between for and lola during multiple developmental processes, including the motor axon guidance of the embryonic nervous system.

\section{PKG physically interacts with LolaT both in vitro and in vivo}

Given the genetic interaction between for and lola, we wondered whether the two proteins PKG and Lola have a physical association. It is known that different Lola isoforms have distinct functions (Goeke et al., 2003; Spletter et al., 2007; Fukui et al., 2012; Southall et al., 2014). Therefore, three relatively well-studied Lola proteins (LolaA, LolaL, and LolaT) were tested for the physical association with PKG by coimmunoprecipitation experiments using Drosophila S2 cells. However, the three Lola isoforms in our study are in accordance with those in Flybase by reason of different naming conventions (Davies et al., 2013). Flag-tagged PKG (Flag-PKG-P2) and each of the three different Myc-tagged Lola isoforms (Myc-LolaA, Myc-LolaL, Myc-LolaT) were cooverexpressed in S2 cells. Interestingly, among the three different Lola proteins pulled down with anti-Myc antibody, only LolaT coimmunoprecipitated with PKG-P2 (Fig. 4A). Reciprocally, immunoprecipitation of Flag-PKG-P2 using anti-Flag antibody also coprecipitated Myc-LolaT (Fig. 4B). Physical association of LolaT with the other two PKG isoforms (P1, P3) in S2 cells was also detected (data not shown). These results indicate that PKG is physically associated with a specific isoform of Lola, LolaT.

To further substantiate the association of PKG with LolaT, particularly to see whether the association exists in Drosophila embryos, we generated UAS-myc-lolaT transgenic flies to express a fused protein of Myc-LolaT in all neurons using the elav-Gal4 driver. In protein extracts from embryos expressing Myc-LolaT, we did not detect coimmunoprecipitation of endogenous PKG 
A $3621 \mathrm{~K}$ $3622 \mathrm{~K}$ $3623 \mathrm{~K}$
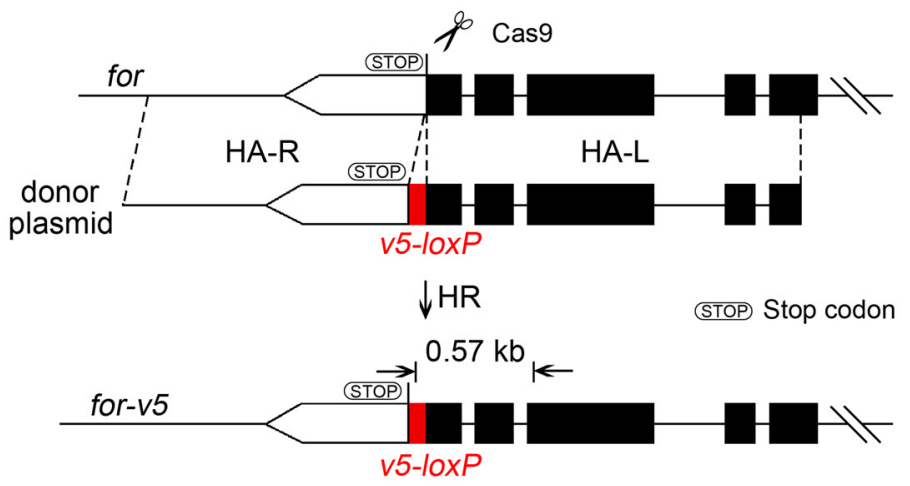

B $0.57 \mathrm{~kb}$

C

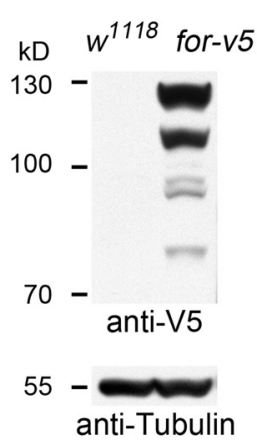

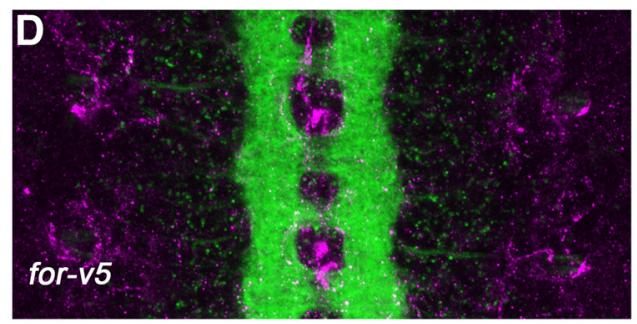

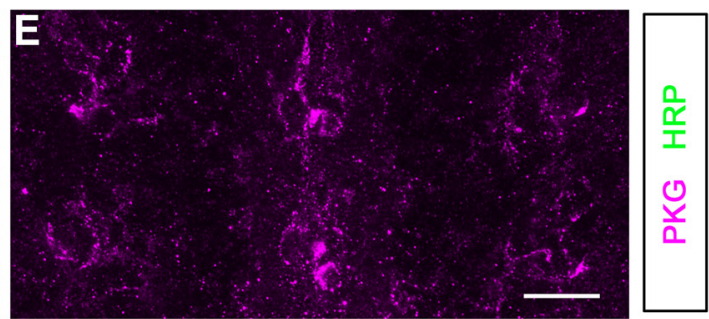

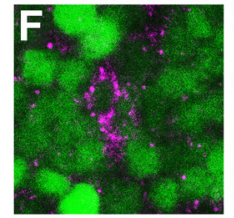
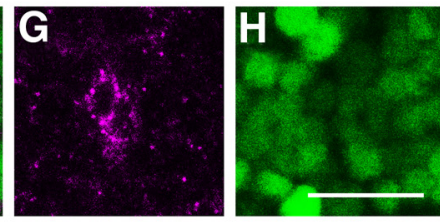

elav-Gal4/+·for-v5/+:UAS-GFP.n/s/+
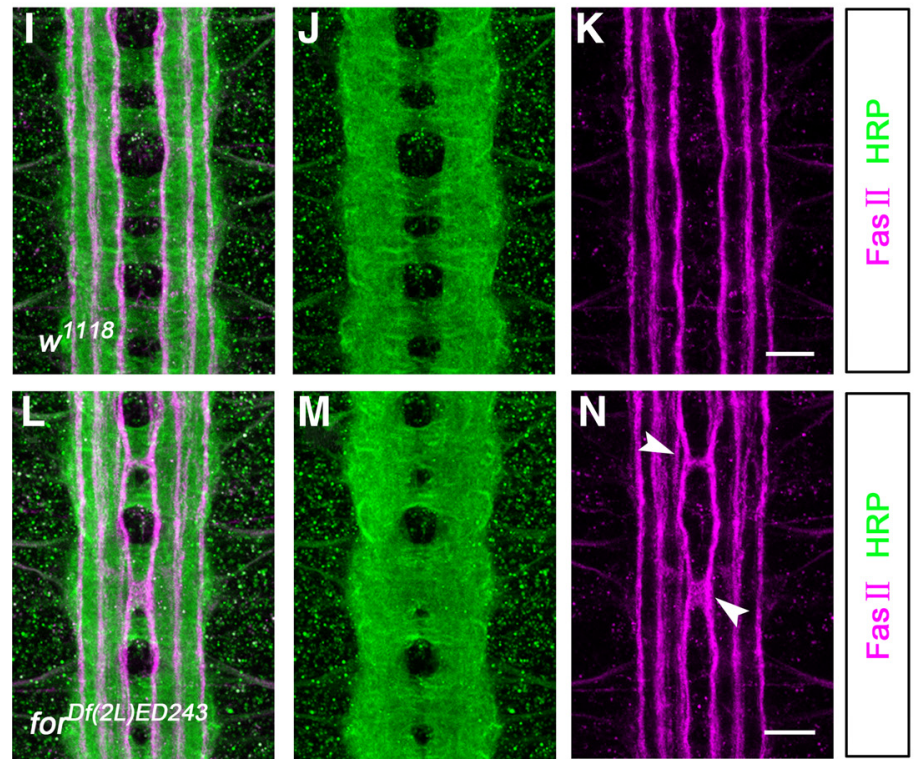

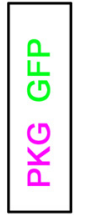

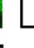

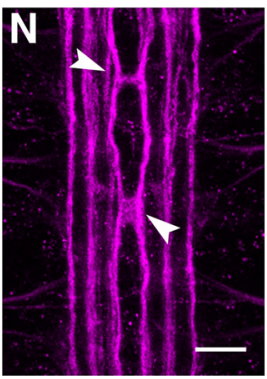

0

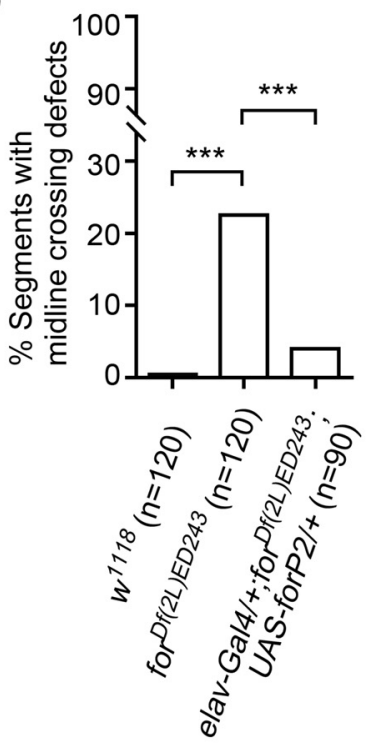

Figure 2. for is expressed in the VNC and is required for CNS axon guidance. A, Schematic diagram of the strategy to generate a for-v5 in vivo tagged line using the CRISPR/Cas9 system. Scissors indicate where the CRISPR/Cas9 cleaves at the for locus. Empty box marked STOP represents the stop codon of the for gene. Filled red box represents the v5-loxP sequence. Dashed lines indicate the homologous regions in the Drosophila genome and the donor plasmid. Two opposite arrows indicate the pair of primers used for PCR identification. $\boldsymbol{B}$, for- 15 was characterized by successful amplification of a $0.57 \mathrm{~kb}$ fragment. $\mathrm{M}$, DNA marker; PC, positive control, the corresponding PCR product amplified from the donor plasmid; NC, negative control, no corresponding PCR product amplified from the $w^{1718}$ genomic DNA. C, Detection of the fused PKG-V5 protein in for-v5 embryos. Protein extracts from $w^{1718}$ or for-v5 embryos were prepared and analyzed with an anti-V5 antibody. Different PKG isoforms are detected in for-v5 embryos. Tubulin is used as a protein loading control. D, E, Limited Z-stacks of the staining results showing two segments of the VNC. PKG-V5 (magenta) is expressed in the VNC at late embryonic Stage 16. CNS axons stained by HRP-FITC (green) are the position markers. Scale bar, $10 \mu \mathrm{m}$. F-H, PKG-V5 (magenta) is detected in a high level in the cytoplasm of the neurons (green). Scale bars, $10 \mu \mathrm{m}$. $\boldsymbol{I - K}$, CNS axons of $w^{1718}$ embryos are visualized with HRP (green) and anti-Fasll antibody (magenta). Scale bars, $10 \mu \mathrm{m} . \boldsymbol{L}-\boldsymbol{N}$, for Pf(2)ED243 homozygous embryos exhibit midline crossing defects ( $\boldsymbol{N}$, arrows). Scale bars, $10 \mu \mathrm{m} . \mathbf{0}, \mathbf{Q}$ Quantification of the midline crossing defects in $w^{1118}$, for ${ }^{D f(2 L) E 243}$ and rescued embryos. $n=$ the number of segments scored for each genotype. ${ }^{* * *} p<0.001$ (Fisher's exact test). 
A

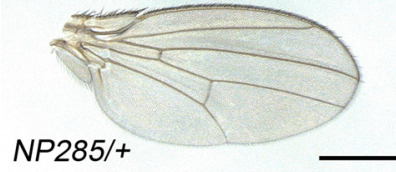

C

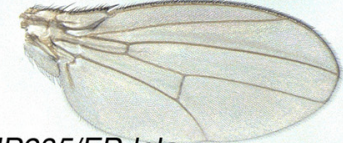

NP285/EP-lola
B

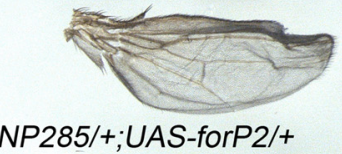

D

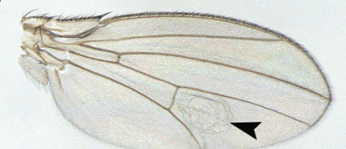

NP285/EP-lola;UAS-forP2/+
E

口normal $\square$ blistered $\boldsymbol{\square}$ balloon

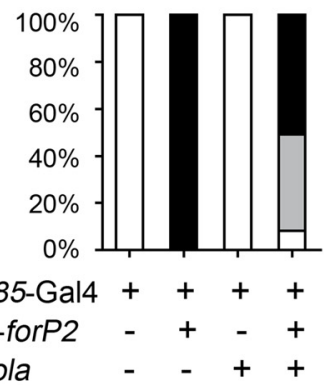

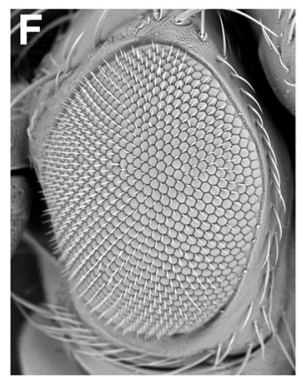
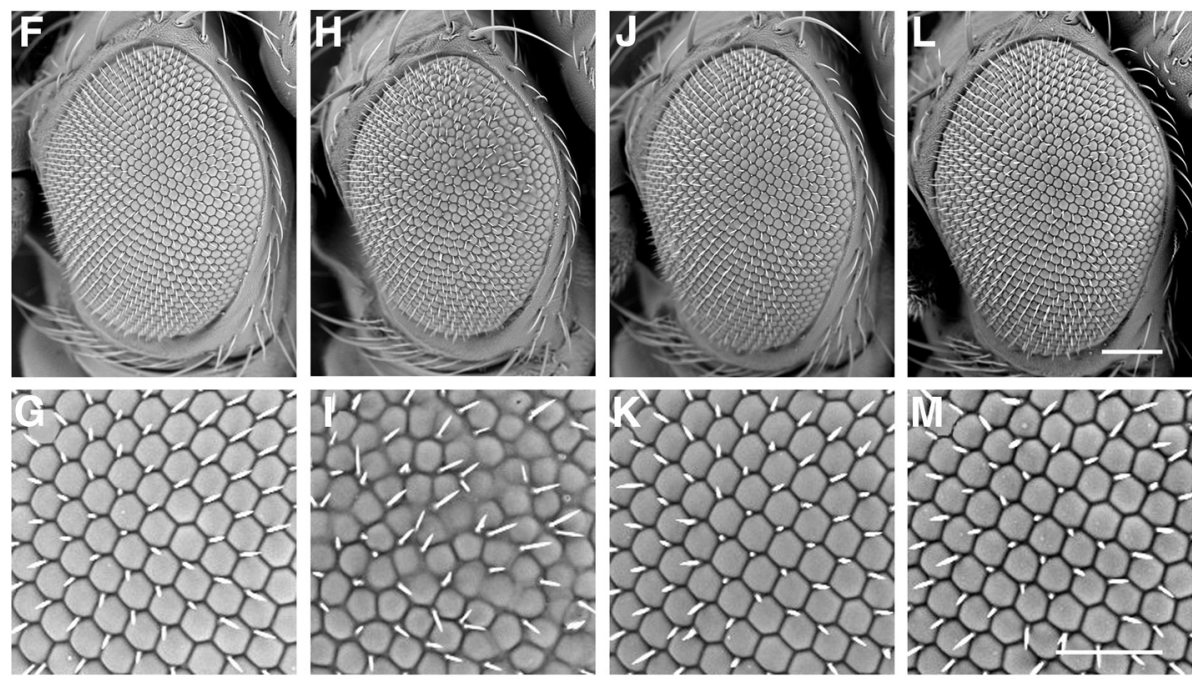

GMR-Gal4/+

GMR-Gal4/+;

$\begin{array}{ll}\text { GMR-Gal4/+; } & \text { GMR-Gal4/+; } \\ \text { UAS-forRNAi[V38320]/+ UAS-lolaRNAi/+ }\end{array}$

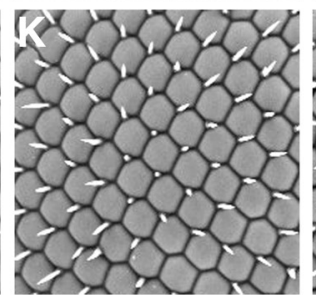

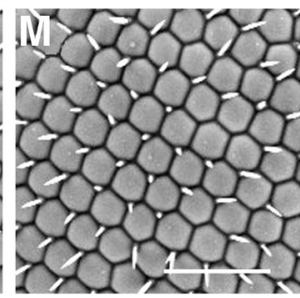

GMR-Gal4/+;

UAS-forRNAi[v38320]/,

UAS-IolaRNAi
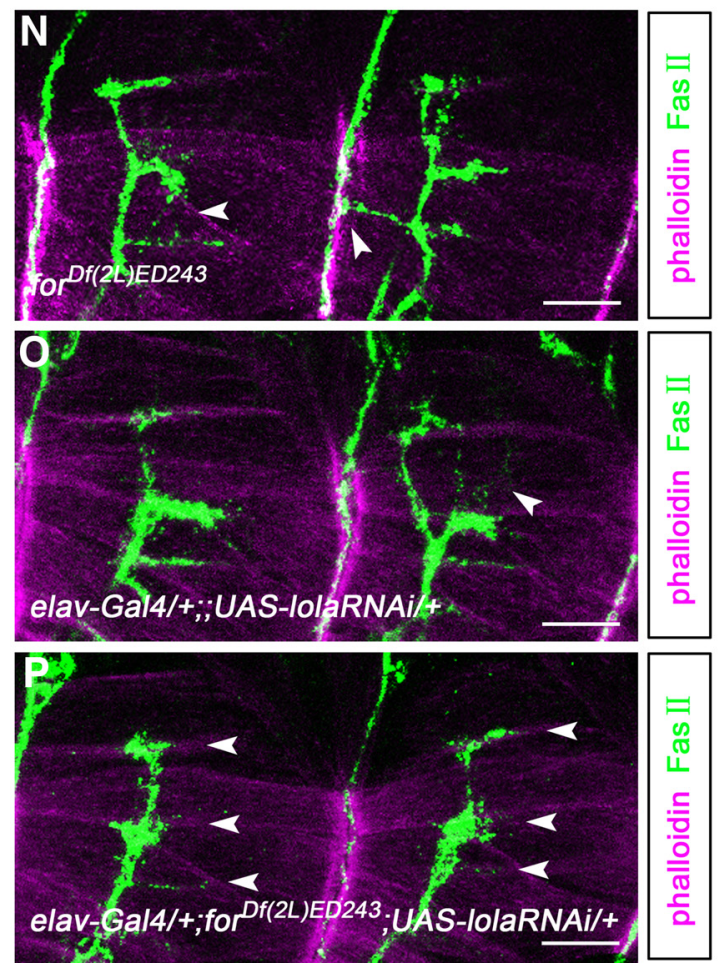

Q
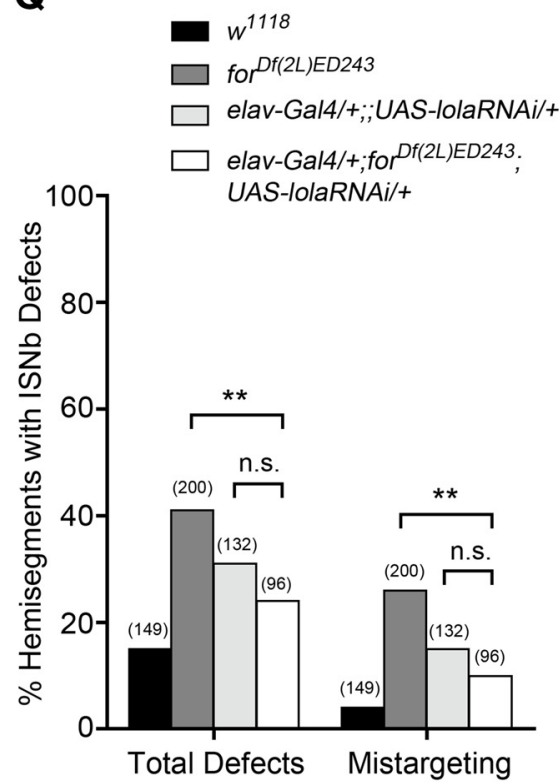

Figure 3. for genetically interacts with lola in wing, eye, and ISNb motor axons. $A-E$, Genetic interaction of for and lola in the wing. $A$, Normal wing of control flies. $B$, Balloon wing caused by overexpression of PKG under the control of NP285-Gal4. C, Normal wing of the flies overexpressing lola. D, Blistered wing partially rescued by coexpression (Figure legend continues.) 
A

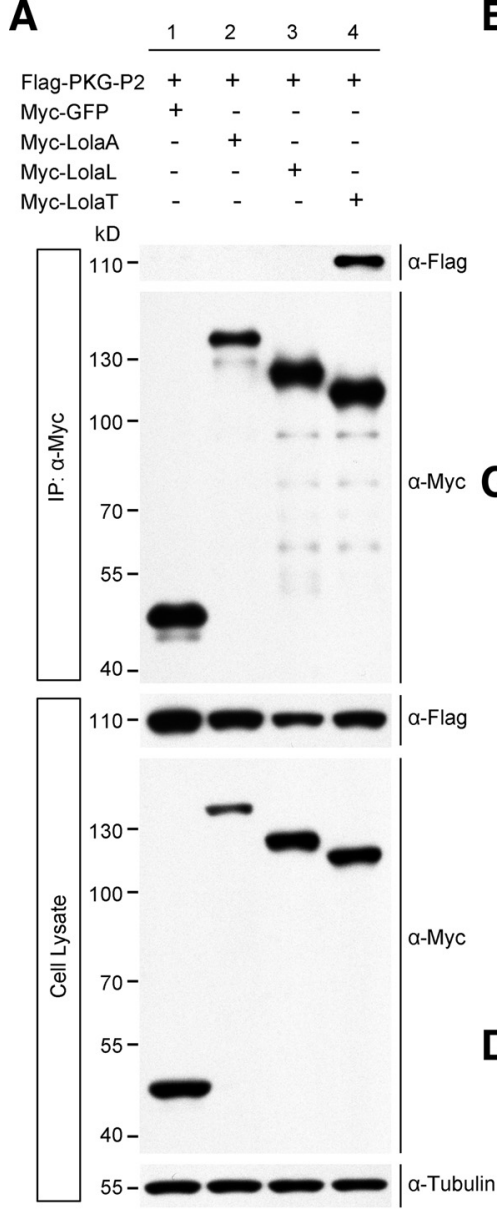

B

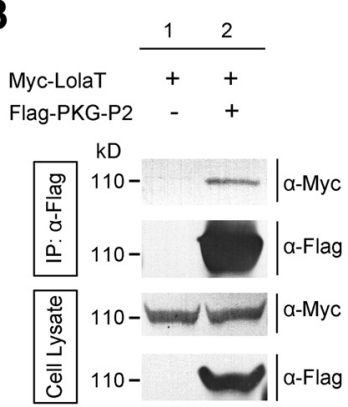

C
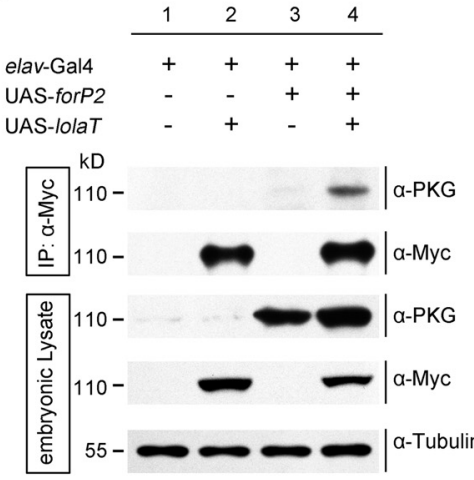

D

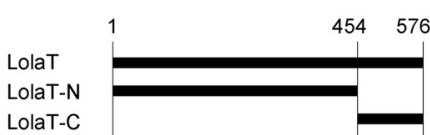

$\mathbf{E}$

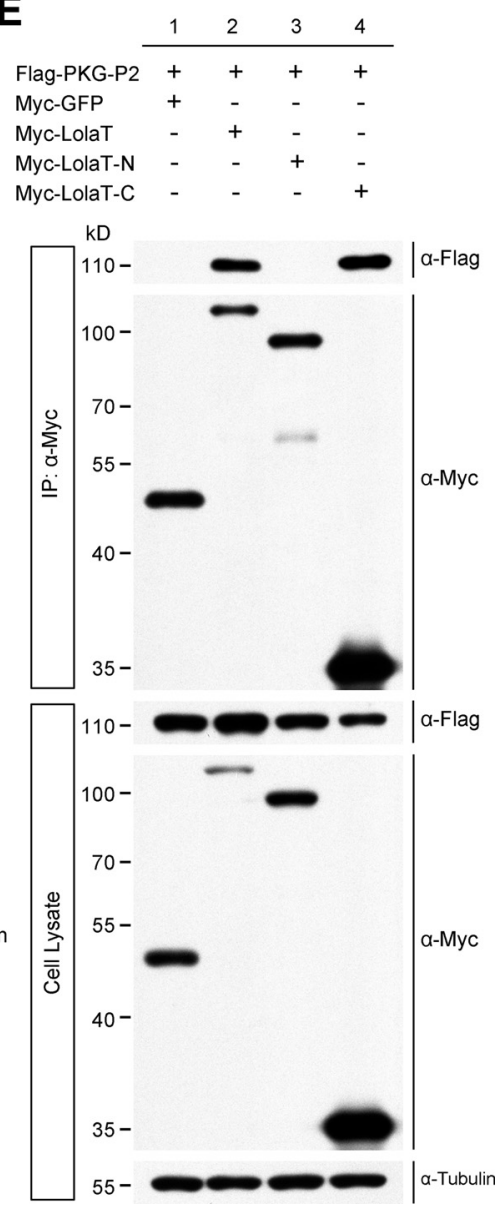

Figure 4. PKG physically interacts with LolaT both in vitro and in vivo. A, PKG-P2 is specifically associated with LolaT in S2 cells. Lysates from Drosophila S2 cells expressing Flag-PKG-P2 and Myc-GFP, Myc-LolaA, Myc-LolaL or Myc-LolaT were used for immunoprecipitation with anti-Myc and blotted with anti-Flag to detect the presence of Flag-PKG-P2 (lane 4). B, PKG-P2 and LolaT physical interact in S2 cells. Lysates from Drosophila S2 cells expressing Myc-LolaT with or without Flag-PKG-P2 were used for immunoprecipitation with anti-Flag antibodies and blotted with anti-Myc to detect the presence of Myc-LolaT (lane 2). C, PKG-P2 physically interacts with LolaT in Drosophila embryos. Lysates from embryos with different genetic backgrounds, expressing PKG-P2 under the control of elav-Gal4 with or without Myc-LolaT, were used for immunoprecipitation with the anti-Myc antibody and blotted with anti-PKG to detect the presence of PKG protein (lane 4). D, Schematic diagram representing the LolaT constructs used for protein interaction assays with PKG-P2. The full-length LolaT protein is 576 amino acids. LolaT-N represents the N-terminal domain that is conserved in all Lola isoforms. LolaT-C represents the specific C-terminal region of the LolaT isoform. $E$, The specific C-terminal region of LolaT is required for the physical interaction between PKG-P2 and LolaT. Lysates from Drosophila S2 cells expressing Flag-PKG-P2 and Myc-GFP, Myc-LolaT, Myc-LolaT-N, or Myc-LolaT-C were used for immunoprecipitation with the anti-Myc antibody and blotted with anti-Flag to detect the presence of Flag-PKG-P2 (lane 2, 4).

(Fig. 4C); however, when both PKG-P2 and Myc-LolaT were expressed in the nervous system of Drosophila embryo, PKG and LolaT were coimmunoprecipitated with the anti-Myc antibody (Fig. 4C). To dissect the regions of LolaT mediating the physical

$\leftarrow$

(Figure legend continued.) of for and lola. E, The penetrance and expressivity of the wing defects for flies with different genotypes. Overexpression of lola partially rescues the defective balloon wing phenotype caused by the overexpressed for. $\boldsymbol{F}-\boldsymbol{M}$, Genetic interaction of for and lola in the eye. $\boldsymbol{F}, \mathbf{G}$, Normal eye of control flies. $\boldsymbol{H}, \boldsymbol{I}$, Rough eye phenotype caused by knocking down of for in the eye.J, $\boldsymbol{K}$, Normal eye of the flies with Iola knocked down in the eye. $L, M$, Rescued eye of the flies with both for and lola knocked down in the eye. $\boldsymbol{N - P}$, ISNb motor axon projections of late Stage 16 embryos visualized with anti-Fasll (green). Muscles are stained with phalloidin (magenta). All panels, Anterior is to the left and dorsal is top. Scale bar, $10 \mu \mathrm{m} . \boldsymbol{N}$, In for P(2LLED243 mutants, ISNb motor axons innervate improper muscles or abnormally associate with adjacent axons (arrows). 0 , ISNb motor axons exhibit extra nerve endings (arrow) when Iola RNAi is expressed in the nervous system. $\boldsymbol{P}$, Axon guidance defects in fo ${ }^{D f(2 L) E D 243}$ are suppressed by lola knockdown in the nervous system. Q, Quantification of total defects and mistargeting ISNb phenotypes in different genetic backgrounds. ISNb total defects and mistargeting defects in for ${ }^{D f(2 L) E D 243}$ are partially suppressed by knockdown of /ola in the nervous system. The number of abdominal hemisegments scored for each genotype is shown in parentheses. ${ }^{* *} p<0.01$ (Fisher's exact test). n.s., Not significant. interaction between LolaT and PKG, we generated two truncated Myc-LolaT constructs: the conserved Lola $\mathrm{N}$-terminal region with the BTB domain (Myc-LolaT-N) and the variable LolaT C-terminal region with the zinc finger domain (Myc-LolaT-C) (Fig. 4D). Each truncated LolaT construct was coexpressed with Flag-PKG-P2 in S2 cells. Interestingly, but not surprisingly, FlagPKG-P2 coimmunoprecipitated with the C-terminal region of LolaT, but not with the N-terminal region, which is conserved in all Lola isoforms (Fig. 4E). These results indicate that PKG-P2 and LolaT physically interact with each other in embryonic neuronal cells, and the interaction is dependent on the C-terminal region of LolaT.

\section{PKG antagonizes LolaT to regulate ISNb axon guidance}

The molecular interaction between LolaT and PKG suggested that lolaT may be involved in for-mediated motor axon guidance. To this end, we generated a lolaT-specific mutant using the CRISPR/Cas9 system. A gRNA-binding site was chosen in the C-terminal region of LolaT, without affecting the other Lola isoforms. lola $T^{38-1}$ containing a 1 bp deletion was generated, which 
A

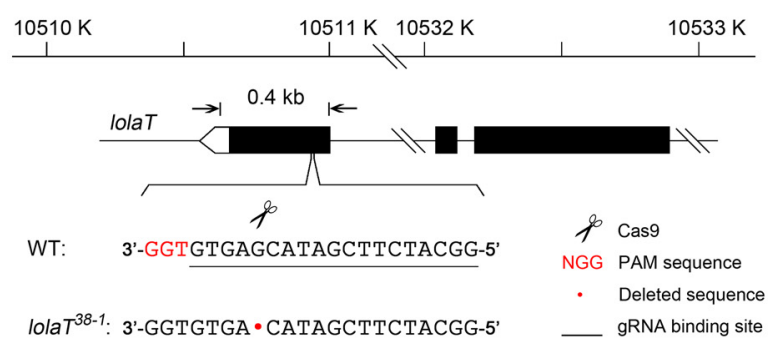

B

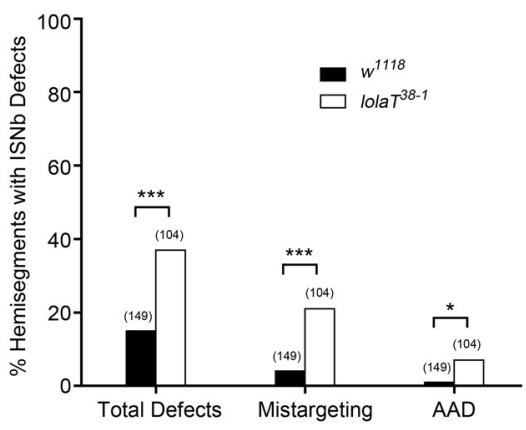

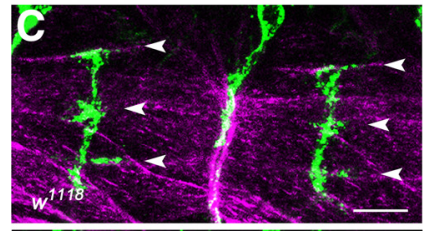

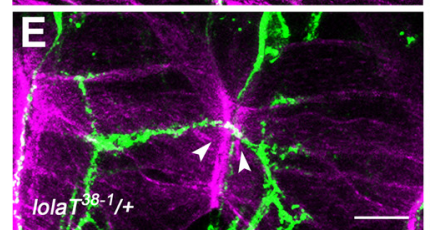

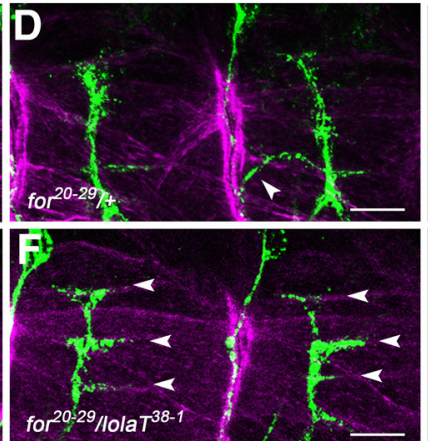
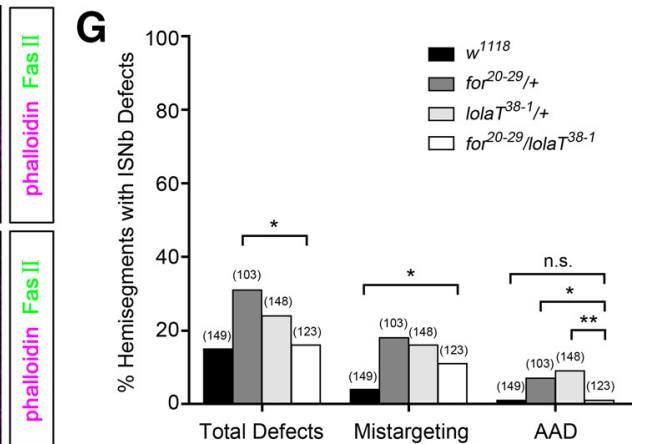

Figure 5. for and lolaT genetically interact to regulate ISNb axon guidance. $A$, Schematic diagram representing the generation and characterization of the $/ 0 / a T^{38-1}$ mutant using the $C R I S P R / C$ as 9 system. Scissors indicate where Cas 9 cleaves in the lolaT locus. The PAM sequence is marked in red. The gRNA binding sequence is underlined. Red dot represents the 1 bp deletion in the 1 olaT genomic sequence. B, Quantification of ISNb total defects, mistargeting defects and AADs in $w^{1718}$ and lola $T^{38-1}$ embryos. AAD, the defects with abnormal associations between ISNb and other axons. $\boldsymbol{C}-\boldsymbol{F}$, ISNb motor axon projections of late Stage 16 embryos visualized with anti-Fasll (green). Muscles are stained with phalloidin (magenta). All panels, Anterior is to the left and dorsal is top. Scale bar, $10 \mu \mathrm{m}$. C, In $w^{1118}$, ISNb motor axons form three kinds of innervations (arrows) with the ventral muscles in each hemisegment. $\boldsymbol{D}$, In heterozygous for ${ }^{20-29}$ mutants, ISNb and transverse (TN) axons make abnormal contacts (arrow). $\boldsymbol{E}$, In the heterozygous /ola $T^{38-1}$ mutant, ISNb axons of two adjacent segments abnormally associate (arrows). $\boldsymbol{F}$, Mutants heterozygous for both for ${ }^{20-29}$ and Iola $T^{38-1}$ exhibit normal ISNb axon projections (arrows). G, Quantification of ISNb total defects, mistargeting defects and AADs in different genetic backgrounds. The percentage of AADs in double-heterozygous for for ${ }^{20-29}$ and lola ${ }^{38-1}$ is significantly decreased compared with each of the two single heterozygous mutants. The number of abdominal hemisegments scored for each genotype is shown in parentheses. ${ }^{*} p<0.05$ (Fisher's exact test). ${ }^{* *} p<0.01$ (Fisher's exact test). n.s., Not significant.

results in a frame shift of the LolaT coding sequence (Fig. 5A). The ISNb motor axon development in lola $T^{38-1}$ homozygous mutants showed similar targeting defects to those observed in the for mutant embryos, especially the defects with abnormal associations with other axons (AAD). Compared with the $w^{1118}$ control, the total defects, mistargeting defects, and the AADs in lola $T^{38-1}$ are significantly increased (Fig. 5B). Next, we examined whether for and lolaT genetically interact with each other in ISNb pathfinding and target recognition. Interestingly, compared with the $w^{1118}$ control (Fig. 5C), both heterozygous for ${ }^{20-29}$ and heterozygous lola $T^{38-1}$ mutants are haplo-insufficient, displaying AADs in ISNb motor axons (Fig. 5D, E, arrows). More importantly, when heterozygous lola $T^{38-1}$ was combined with heterozygous for ${ }^{20-29}$, the ISNb axon guidance defect phenotype was suppressed (Fig. $5 F$ ). Total defects of ISNb axons in the double-heterozygous embryos were significantly decreased compared with the heterozygous for ${ }^{20-29}$ mutants (Fig. 5G). Moreover, the AADs of ISNb axons in the double-heterozygous embryos were rescued (Fig. $5 G$ ). These results suggest that ISNb abnormally associated with adjacent axons is controlled by an antagonistic effect between for and lolaT.

\section{PKG regulates the cellular localization of LolaT}

lola encodes diverse BTB-Zn-finger transcription factors (Spletter et al., 2007) that regulate transcription of a set of specific genes (Cavarec et al., 1997; Gates et al., 2011). To investigate how PKG regulates the function of LolaT, we examined the cellular localization of PKG-P2 and LolaT in S2 cells by immunostaining.
When Myc-LolaT was expressed in S2 cells alone, most LolaT proteins were located in the nucleus, as detected by an Myc-tag antibody (Fig. 6A-D). However, LolaT proteins were restricted in the cytoplasm of $23.58 \%$ S2 cells with PKG-P2 and LolaT coexpressed (Fig. $6 E-H$ ). The percentage of the cytoplasmic retention of LolaT was significantly increased when PKG-P2 and LolaT were coexpressed in S2 cells compared with cells only expressing LolaT (Fig. 6I). In the observation, the subcellular localization of PKG did not significantly change upon coexpression with LolaT. In addition, LolaA and LolaL were not significantly affected by PKG-P2 in S2 cells as assayed by staining experiments (data not shown). Next, to test whether the LolaT protein without the specific $\mathrm{C}$ terminus is not affected by PKG, we performed experiments with LolaT-N and PKG-P2 coexpressed in S2 cells. As expected, LolaT-N proteins were always detected in the nucleus (Fig. $6 J-M$ ).

To exclude the possibility that the cytoplasmic retention of LolaT is caused by the breakdown of the nuclear membrane, we introduced hydroxyurea to block DNA biosynthesis in S2 cells to inhibit cell division (Heinemann et al., 2010). Flow cytometry was performed to analyze the cell cycle distribution. Without hydroxyurea treatment, the cell cycle distributions of $\mathrm{S} 2$ cells in $\mathrm{G}_{1}, \mathrm{~S}$, and $\mathrm{G}_{2}$ phase were $30.43 \%, 28.53 \%$, and $41.04 \%$, respectively. When S2 cells were treated with $1 \mathrm{~mm}$ hydroxyurea for $18 \mathrm{~h}$, the cell cycle distributions of $\mathrm{S} 2$ cells in $\mathrm{G}_{1}, \mathrm{~S}$, and $\mathrm{G}_{2}$ phase came out to be $48.94 \%, 47.22 \%$, and $3.83 \%$, respectively. The significantly decreased percentage of $G_{2}$ cells indicates that few cells were entering the $M$ phase. Under this condition of hy- 

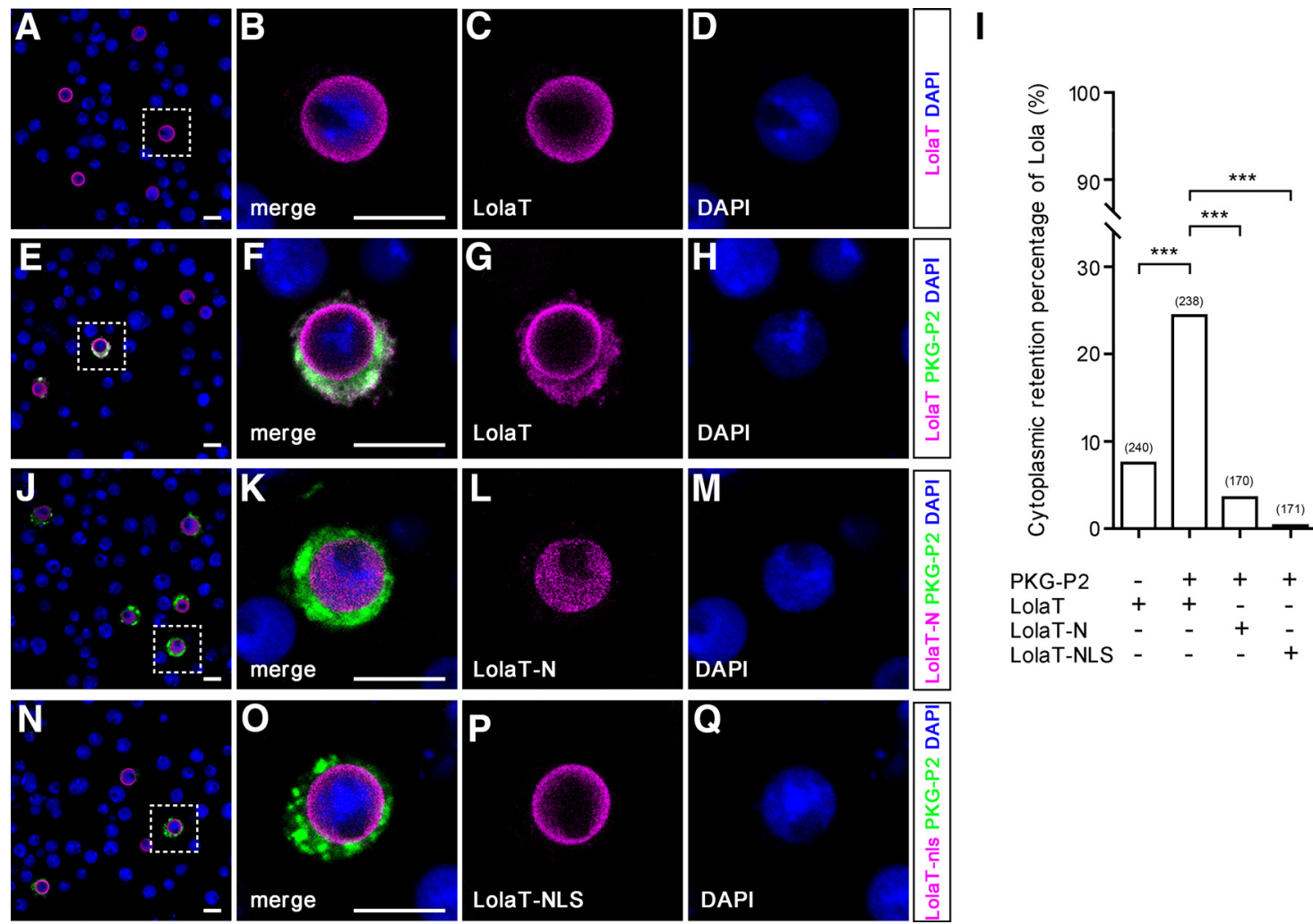

$\begin{array}{lcccc}\text { PKG-P2 } & - & + & + & + \\ \text { LolaT } & + & + & - & - \\ \text { LolaT-N } & - & - & + & - \\ \text { LolaT-NLS } & - & - & - & +\end{array}$

Figure 6. PKG sequesters LolaT in the cytoplasm in S2 cells. $\boldsymbol{A}-\boldsymbol{H}, \mathrm{S} 2$ cells transfected with Myc-LolaT alone $(\boldsymbol{A}-\boldsymbol{D})$ or in combination with Flag-PKG-P2 (E-H) were stained with anti-Myc antibody and anti-Flag antibody. DNA is visualized with DAPI. Scale bars, $10 \mu \mathrm{m}$. $\boldsymbol{A}$, Overview of $\mathbf{2}$ cells with Myc-LolaT transfection alone. Dashed box represents the cell in high-magnification images (B-D). $\boldsymbol{B}-\boldsymbol{D}$, LolaT is detectable in the nucleus when only LolaT is transfected. $\boldsymbol{E}$, Overview of S2 cells with Myc-LolaT and Flag-PKG-P2 cotransfection. Dashed box represents the cell in high-magnification images $(\boldsymbol{F}-\boldsymbol{H}) . \boldsymbol{F}-\boldsymbol{H}$, LolaT is retained in the cytoplasm and colocalizes with PKG-P2 in the S2 cells overexpressing both PKG-P2 and LolaT. I, The cytoplasmic retention percentage of different Lola proteins in the transfected S2 cells. $n=$ the number of scored S2 cells. ${ }^{* * *} p<0.001$ (Fisher's exact test). J-Q, S2 cells cotransfected with Flag-PKG-P2 and different Lola proteins were stained with anti-Myc antibody and anti-Flag antibody. DNA is visualized with DAPI. Scale bars, $10 \mu \mathrm{m}$.J, Overview of S2 cells with Myc-LolaT-N and Flag-PKG-P2 cotransfection. Dashed box represents the cell in high-magnification images $(\boldsymbol{K}-\boldsymbol{M}) . \boldsymbol{K}-\boldsymbol{M}$, LolaT-N is in the nucleus of the $\mathbf{S 2}$ cells overexpressing both PKG-P2 and LolaT-N. $\boldsymbol{N}$, Overview of S2 cells with Myc-LolaT-NLS and Flag-PKG-P2 cotransfection. Dashed box represents the cell in high-magnification images (0-Q). $\mathbf{0}-\mathbf{Q}$, LolaT-NLS is in the nucleus of the S2 cells overexpressing both PKG-P2 and LolaT-NLS.

droxyurea treatment, the percentage of the cells containing cytoplasmic LolaT still significantly increased in the cells with both LolaT and PKG overexpressed (16.36\%), compared with the cells with only LolaT overexpressed (2.54\%). To discern the mechanism underlying the cytoplasmic retention of LolaT, we added a strong, constitutive NLS to the C terminus of Myc-LolaT. We found that LolaT-NLS proteins were always detected in the nucleus, although both PKG and LolaT-NLS were overexpressed in S2 cells (Fig. 6I, N-Q). These results suggest a mechanism by which PKG regulates the function of LolaT by altering its subcellular localization.

\section{Discussion}

PKG is an important intracellular effector of the cGMP signaling pathway, which has various functions, including neuronal plasticity, learning, and memory (Schmidt et al., 2002; Kaun et al., 2007; Wang et al., 2008; Reaume and Sokolowski, 2009; Francis et al., 2010). Our previous studies have revealed that for is required for visual pattern memory in the central complex of Drosophila (Wang et al., 2008). Other studies reported that for $^{S}$ and for ${ }^{22}$ larvae, which have reduced PKG activity, have increased ectopic nerve endings in the neuromuscular junction of ISNb motor neurons (Renger et al., 1999), but the precise roles of PKG in neuronal circuit formation remain to be clarified. In this work, we show that neuronal PKG is required for regulating axon guidance in Drosophila embryonic stages by antagonizing the effects of LolaT.
PKG and LolaT physically interact with each other both in vitro and in vivo, an interaction that is dependent on the variable C-terminal region of LolaT. At the molecular level, PKG modulates the nucleo-cytoplasmic localization of LolaT in S2 cells. Our findings suggest opposing functions of PKG and LolaT in regulating axon guidance.

The Drosophila neuromuscular system is a good model to investigate the role and the underlying mechanism of PKG in axon guidance. The stereotypic morphology of the ISNb motor axon from A2-A6 in late embryonic Stage 16 was selected for analysis in this study. In the for ${ }^{D f(2 L) E D 243}$ hypomorphic mutant and the $f o r^{20-29}$ null mutant, ISNb axons exhibit a similar proportion of mistargeting defects ( $26 \%$ and $31.41 \%$, respectively), which is significantly higher than that of $w^{1118}$ control flies. Surprisingly, but importantly, the heterozygous for ${ }^{20-29}$ mutants also showed ISNb mistargeting defects of $18.45 \%$, which provide a basis for genetic interaction tests. In addition to the mistargeting defects that we have focused on in our study, other ISNb abnormalities, such as stalled, bypass, or premature defasciculation, were also rarely observed in both the for mutants and the $w^{1118}$ control. In the CNS, the midline crossing defects in the for ${ }^{D f(2 L) E D 243}$ mutant suggest a general role of for in the nervous system during development.

for ${ }^{D f(2 L) E D 243}$ is homozygous viable until late larval stages but is pupal lethal (Belay et al., 2007). In our study, the for ${ }^{20-29}$ null mutants we generated are also pupal lethal. The pupal lethality 
can be attributed to a possible maternal effect of the for gene, which may also account for the relatively low penetrance of the described axon guidance targeting defects in this study. Hence, we examined the protein level of PKG in the embryonic stages of $f o r^{20-29}$ and for ${ }^{D f(2 L) E D 243}$ mutants. Indeed, a gradual decrease of PKG was detected as embryo development proceeded (data not shown), suggesting the possibility of a maternal effect.

PKG is ubiquitously expressed throughout the embryonic stages. At Stage 15, PKG is still expressed ubiquitously but with stronger signal intensity in the nervous system. As the VNC condensation and segmental differentiation, PKG starts to attain a segmental distribution in the VNC at Stage 16. The enrichment of PKG in each segment of the VNC becomes even more pronounced at Stage 17 (data not shown). PKG was detected with a high level in the cytoplasm of the neurons (Fig. $2 \mathrm{~F}-\mathrm{H}$ ), but it is also detected in other types of cells (data not shown). We also tried to label the glial cells in the late embryonic stages using Repo-Gal4, however, without success. Thus, it is not certain yet whether PKG is specifically enriched in the glial cells. The RP motor neurons are located in the midline of the VNC, which can be distinguished according to their position (Sink and Whitington, 1991a,b). According to the observation, PKG is likely enriched in the RP motor neurons. However, it is not conclusive at this moment because we do not have proper RP motor neuron Gal4 lines or other markers to specifically label the RP motor neurons.

lola is known as a master regulator of axon guidance of ISNb motor neurons in Drosophila (Madden et al., 1999). Nevertheless, how Lola is regulated was still not clear. We show that knockdown of lola in the nervous system suppresses the ISNb targeting defects of $f o r^{D f(2 L) E D 243}$. lola encodes a family of BTB-Zn-finger transcription factors with at least 20 different protein isoforms (Spletter et al., 2007). Each Lola isoform contains a conserved common $\mathrm{N}$-terminal BTB domain and a variable $\mathrm{C}$-terminal specific Zn-finger domain (Goeke et al., 2003; Horiuchi et al., 2003; Ohsako et al., 2003). Lola isoforms have distinct functions in axon guidance (Goeke et al., 2003). Thus, we hypothesized that PKG may cooperate with only one or a few of the specific Lola isoforms in regulating ISNb pathfinding and target recognition. Coimmunoprecipitation analyses in S2 cells were performed between PKG and different Lola isoforms. Indeed, one specific isoform of Lola, LolaT, was detected to interact with PKG. A previous study reported that ISNb peripheral nerve fails to form connections to their cognate muscles in strong lola mutants $\left(\right.$ lola $^{5 \mathrm{DD}}$, lola ${ }^{1 \mathrm{~A} 4}$ ) or in embryos with overexpression of lola4.7 (lola-RR and lola-RG in Flybase) (Madden et al., 1999), indicating a requirement for the correct level of lola expression. In our study, either loss of lolaT or overexpression of lolaT (data not shown) in the nervous system caused ISNb abnormally associated with other motor axons, suggesting a dose-dependent function of lolaT in motor axon pathfinding and target recognition. The different phenotypes of ISNb axons between lolaT mutants and lola null mutants indicate the specific function of different lola transcripts. Embryos heterozygous for both lola ${ }^{38-1}$ and for $^{20-29}$ exhibit normal ISNb motor axon guidance behavior, suggesting that PKG may have a key role in regulating the functional dosage of LolaT. Consistently, overexpression of LolaT in the nervous system in a for ${ }^{20-29}$ heterozygous mutant background did not lead to more severe ISNb axon guidance defects than those in a wild-type background (data not shown). The antagonistic mechanism between PKG and LolaT provides an important strategy for axon guidance during embryonic development.
It is important to detect the endogenous colocalization of PKG and Lola in the embryo to further explore the mechanism in $v i v o$. Using anti-Lola antibody, we detected that Lola is expressed in almost all the nuclei of the nervous system of late Stage 16 embryos (data not shown). The cells with high expression level of PKG, shown in Figure 2, $D$ and $E$, show a colocalization of PKG with Lola (data not shown). However, both PKG and Lola are undetectable in axons (data not shown). We also used elav-Gal4 to overexpress LolaT-Myc in the nervous system. As expected, overexpressed LolaT appeared in the nuclei of the neurons (data not shown). Therefore, our results indicate a model that PKG is not strongly localized to axons and growth cones during periods of motor and CNS axonal pathfinding but is situated within the cell body where it locally regulates the access of LolaT to the nucleus. However, the mechanism between PKG and Lola remains to be illuminated in vivo and in neurons.

Transcription factors containing an NLS can be recognized by karyopherins directly or by importin $\alpha$ in the presence of an adaptor protein (Goldfarb et al., 2004; Mosammaparast and Pemberton, 2004). Our results show that LolaT accumulates in the cytoplasm of some S2 cells (23.58\%) with PKG and LolaT cotransfected. However, a low percentage of cells (8.06\%) transfected with only LolaT also show the cytoplasmic localization of LolaT, which might be attributed to the endogenous PKG in S2 cells. PKG is mainly localized in the cytoplasm; therefore, it would be reasonable to hypothesize that the interaction between PKG and LolaT prevents the nuclear import of LolaT. This hypothesis is supported by another result that LolaT-NLS protein is no longer restrained in the cytoplasm by the overexpressed PKG.

It is known that phosphorylation can either promote or inhibit the nuclear import of transcription factors, but few cargos have been characterized to date (Nardozzi et al., 2010). A few phosphorylation targets of PKG I in mammals have been reported (Francis et al., 2010); however, no such protein in Drosophila has yet been discovered. NetPhosK analyses revealed several predicted phosphorylation sites of PKG in LolaT, supporting LolaT to be a phosphorylatable substrate of PKG. We propose two possible mechanisms of how PKG regulates the nuclear import of LolaT. One is that LolaT is phosphorylated by $\mathrm{PKG}$, causing LolaT to dissociate from karyopherins, thereby enabling accumulation in the cytoplasm. The other is that the protein-protein interaction between PKG and LolaT impedes the nuclear import of LolaT. Future investigations will focus on clarifying the PKG portions mediating the interaction with LolaT, the PKG phosphorylation site on LolaT, and will screen for more components that function together with PKG-LolaT in determining axon guidance during Drosophila embryonic development.

\section{References}

Ayoob JC, Yu HH, Terman JR, Kolodkin AL (2004) The Drosophila receptor guanylyl cyclase Gyc76C is required for semaphorin-la-plexin A-mediated axonal repulsion. J Neurosci 24:6639-6649. CrossRef Medline

Belay AT, Scheiner R, So AK, Douglas SJ, Chakaborty-Chatterjee M, Levine JD, Sokolowski MB (2007) The foraging gene of Drosophila melanogaster: spatial-expression analysis and sucrose responsiveness. J Comp Neurol 504:570-582. CrossRef Medline

Cavarec L, Jensen S, Casella JF, Cristescu SA, Heidmann T (1997) Molecular cloning and characterization of a transcription factor for the copia retrotransposon with homology to the BTB-containing Lola neurogenic factor. Mol Cell Biol 17:482-494. CrossRef Medline

Chak K, Kolodkin AL (2014) Function of the Drosophila receptor guanylyl cyclase Gyc76C in PlexA-mediated motor axon guidance. Development 141:136-147. CrossRef Medline

Crowner D, Madden K, Goeke S, Giniger E (2002) Lola regulates midline 
crossing of CNS axons in Drosophila. Development 129:1317-1325. Medline

Davies EL, Lim JG, Joo WJ, Tam CH, Fuller MT (2013) The transcriptional regulator lola is required for stem cell maintenance and germ cell differentiation in the Drosophila testis. Dev Biol 373:310-321. CrossRef Medline

Dickson BJ (2002) Molecular mechanisms of axon guidance. Science 298: 1959-1964. CrossRef Medline

Ferres-Marco D, Gutierrez-Garcia I, Vallejo DM, Bolivar J, Gutierrez-Aviño FJ, Dominguez M (2006) Epigenetic silencers and Notch collaborate to promote malignant tumours by $R b$ silencing. Nature 439:430-436. CrossRef Medline

Francis SH, Busch JL, Corbin JD, Sibley D (2010) cGMP-dependent protein kinases and cGMP phosphodiesterases in nitric oxide and cGMP action. Pharmacol Rev 62:525-563. CrossRef Medline

Fukui A, Inaki M, Tonoe G, Hamatani H, Homma M, Morimoto T, Aburatani H, Nose A (2012) Lola regulates glutamate receptor expression at the Drosophila neuromuscular junction. Biol Open 1:362-375. CrossRef Medline

Gates MA, Kannan R, Giniger E (2011) A genome-wide analysis reveals that the Drosophila transcription factor Lola promotes axon growth in part by suppressing expression of the actin nucleation factor Spire. Neural Dev 6:37. CrossRef Medline

Giniger E, Tietje K, Jan LY, Jan YN (1994) lola encodes a putative transcription factor required for axon growth and guidance in Drosophila. Development 120:1385-1398. Medline

Giot L, Bader JS, Brouwer C, Chaudhuri A, Kuang B, Li Y, Hao YL, Ooi CE, Godwin B, Vitols E, Vijayadamodar G, Pochart P, Machineni H, Welsh M, Kong Y, Zerhusen B, Malcolm R, Varrone Z, Collis A, Minto M, et al. (2003) A protein interaction map of Drosophila melanogaster. Science 302:1727-1736. CrossRef Medline

Goeke S, Greene EA, Grant PK, Gates MA, Crowner D, Aigaki T, Giniger E (2003) Alternative splicing of lola generates 19 transcription factors controlling axon guidance in Drosophila. Nat Neurosci 6:917-924. CrossRef Medline

Goldfarb DS, Corbett AH, Mason DA, Harreman MT, Adam SA (2004) Importin alpha: a multipurpose nuclear-transport receptor. Trends Cell Biol 14:505-514. CrossRef Medline

Guo A, Li L, Xia SZ, Feng CH, Wolf R, Heisenberg M (1996) Conditioned visual flight orientation in Drosophila: dependence on age, practice, and diet. Learn Mem 3:49-59. CrossRef Medline

Heinemann L, Simpson GR, Annels NE, Vile R, Melcher A, Prestwich R, Harrington KJ, Pandha HS (2010) The effect of cell cycle synchronization on tumor sensitivity to reovirus oncolysis. Mol Ther 18:2085-2093. CrossRef Medline

Horiuchi T, Giniger E, Aigaki T (2003) Alternative trans-splicing of constant and variable exons of a Drosophila axon guidance gene, lola. Genes Dev 17:2496-2501. CrossRef Medline

Kaun KR, Hendel T, Gerber B, Sokolowski MB (2007) Natural variation in Drosophila larval reward learning and memory due to a cGMP-dependent protein kinase. Learn Mem 14:342-349. CrossRef Medline

Kohn NR, Reaume CJ, Moreno C, Burns JG, Sokolowski MB, Mery F (2013) Social environment influences performance in a cognitive task in natural variants of the foraging gene. PLoS One 8:e81272. CrossRef Medline

Kolodkin AL, Tessier-Lavigne M (2011) Mechanisms and molecules of neuronal wiring: a primer. Cold Spring Harb Perspect Biol 3:piia001727. CrossRef Medline

Landgraf M, Bossing T, Technau GM, Bate M (1997) The origin, location, and projections of the embryonic abdominal motorneurons of Drosophila. J Neurosci 17:9642-9655. Medline

Lee HK, Wright AP, Zinn K (2009) Live dissection of Drosophila embryos: streamlined methods for screening mutant collections by antibody staining. J Vis Exp 34:pii1647. CrossRef Medline

Madden K, Crowner D, Giniger E (1999) lola has the properties of a master regulator of axon-target interaction for SNb motor axons of Drosophila. Dev Biol 213:301-313. CrossRef Medline

Mosammaparast N, Pemberton LF (2004) Karyopherins: from nuclear- transport mediators to nuclear-function regulators. Trends Cell Biol 14: 547-556. CrossRef Medline

Nardozzi JD, Lott K, Cingolani G (2010) Phosphorylation meets nuclear import: a review. Cell Commun Signal 8:32. CrossRef Medline

Nishiyama M, Hoshino A, Tsai L, Henley JR, Goshima Y, Tessier-Lavigne M, Poo MM, Hong K (2003) Cyclic AMP/GMP-dependent modulation of $\mathrm{Ca}^{2+}$ channels sets the polarity of nerve growth-cone turning. Nature 423:990-995. CrossRef Medline

Ohsako T, Horiuchi T, Matsuo T, Komaya S, Aigaki T (2003) Drosophila lola encodes a family of BTB-transcription regulators with highly variable C-terminal domains containing zinc finger motifs. Gene 311:59-69. CrossRef Medline

Osborne KA, Robichon A, Burgess E, Butland S, Shaw RA, Coulthard A, Pereira HS, Greenspan RJ, Sokolowski MB (1997) Natural behavior polymorphism due to a cGMP-dependent protein kinase of Drosophila. Science 277:834-836. CrossRef Medline

Patel NH (1994) Imaging neuronal subsets and other cell types in wholemount Drosophila embryos and larvae using antibody probes. Methods Cell Biol 44:445-487. CrossRef Medline

Reaume CJ, Sokolowski MB (2009) cGMP-dependent protein kinase as a modifier of behaviour. Handb Exp Pharmacol 191:423-443. CrossRef Medline

Renger JJ, Yao WD, Sokolowski MB, Wu CF (1999) Neuronal polymorphism among natural alleles of a cGMP-dependent kinase gene, foraging, in Drosophila. J Neurosci 19:RC28. Medline

Ryder E, Blows F, Ashburner M, Bautista-Llacer R, Coulson D, Drummond J, Webster J, Gubb D, Gunton N, Johnson G, O'Kane CJ, Huen D, Sharma P, Asztalos Z, Baisch H, Schulze J, Kube M, Kittlaus K, Reuter G, Maroy P, et al. (2004) The DrosDel collection: a set of $P$-element insertions for generating custom chromosomal aberrations in Drosophila melanogaster. Genetics 167:797-813. CrossRef Medline

Schmidt H, Werner M, Heppenstall PA, Henning M, Moré MI, Kuhbandner S, Lewin GR, Hofmann F, Feil R, Rathjen FG (2002) cGMP-mediated signaling via cGKIalpha is required for the guidance and connectivity of sensory axons. J Cell Biol 159:489-498. CrossRef Medline

Shelly M, Lim BK, Cancedda L, Heilshorn SC, Gao H, Poo MM (2010) Local and long-range reciprocal regulation of cAMP and cGMP in axon/dendrite formation. Science 327:547-552. CrossRef Medline

Sink H, Whitington PM (1991a) Pathfinding in the central nervous system and periphery by identified embryonic Drosophila motor axons. Development 112:307-316. Medline

Sink H, Whitington PM (1991b) Location and connectivity of abdominal motoneurons in the embryo and larva of Drosophila melanogaster. J Neurobiol 22:298-311. CrossRef Medline

Song H, Ming G, He Z, Lehmann M, McKerracher L, Tessier-Lavigne M, Poo M (1998) Conversion of neuronal growth cone responses from repulsion to attraction by cyclic nucleotides. Science 281:1515-1518. CrossRef Medline

Southall TD, Davidson CM, Miller C, Carr A, Brand AH (2014) Dedifferentiation of neurons precedes tumor formation in lola mutants. Dev Cell 28:685-696. CrossRef Medline

Spletter ML, Liu J, Liu J, Su H, Giniger E, Komiyama T, Quake S, Luo L (2007) Lola regulates Drosophila olfactory projection neuron identity and targeting specificity. Neural Dev 2:14. CrossRef Medline

Wang Z, Pan Y, Li W, Jiang H, Chatzimanolis L, Chang J, Gong Z, Liu L (2008) Visual pattern memory requires foraging function in the central complex of Drosophila. Learn Mem 15:133-142. CrossRef Medline

Yu Z, Chen H, Liu J, Zhang H, Yan Y, Zhu N, Guo Y, Yang B, Chang Y, Dai F, Liang X, Chen Y, Shen Y, Deng WM, Chen J, Zhang B, Li C, Jiao R (2014) Various applications of TALEN- and CRISPR/Cas9-mediated homologous recombination to modify the Drosophila genome. Biol Open 3: 271-280. CrossRef Medline

Zarin AA, Asadzadeh J, Labrador JP (2014) Transcriptional regulation of guidance at the midline and in motor circuits. Cell Mol Life Sci 71: 419-432. CrossRef Medline 